\title{
Design Practice of Ceramic Tea Set Based on Aesthetic Features of Fractal Pattern
}

\author{
Wenming Liu*, Penghui Chen \\ School of Design and Art, Shenyang Jianzhu University, Shenyang, Liaoning, 110168, China
}

\begin{abstract}
The unique aesthetic characteristics of the fractal pattern are analyzed and discussed, and the aesthetic features of the fractal pattern are used to practice the appearance design and decorative pattern design of the ceramic tea set. Through the practice of product design based on fractal ideas, a set of effective and feasible ideas and methods for applying fractal ideas to product design were explored, which broadened product design ideas and tried new possibilities for product design.
\end{abstract}

\section{Fractal-related concepts and their aesthetic characteristics}

Fractal geometry is known as "the geometry of nature", and mathematicians use it as a geometric tool to describe the formation of natural forms. Any complex natural form can be simulated by treating generators as "genes" inherent in complex natural structures, and the final state can be determined by controlling the number of iterations through parameters. Therefore, we can see that fractal geometry can reveal the original appearance and inner structure of nature more realistically. If euclidean geometry studies the geometry of regular graphics, then fractal geometry studies the geometry of "irregular" graphics.

\subsection{Definition of fractal}

The founder of fractal theory, American mathematician B. Mandelbrot, gave a definition of "fractal" in 1982: "A fractal is by definition a set for which the HausdorffBesicoritch dimension strictly exceeds the topological dimension".

In 1986, Mandelbrot gave another definition of fractal "A fractal is a shape made of parts similar to the whole in some way", that is, the fractal has some shape features that are partially similar to the whole in some way [2]. This definition only emphasizes some selfsimilarity of the fractal set, but it is still not comprehensive. In this way, after a long test of theory and application, people still cannot include such a rich fractal world with a concise and general definition.

\subsection{Features of fractal patterns}

The most significant feature of a fractal pattern is that it has a form of self-similarity. It can be regarded as a symmetry that crosses different scales, and contains the recursion of the pattern. The self-similarity may be both approximate self-similarity and statistically. For the meaning of "self-similarity", a figure itself can be regarded as composed of many similar and different parts. For example, Polish mathematician Wactaw Franciszek Sierpinski (1882-1969) proposed a Sierpinski triangle named after himself. The Sierpinski triangle is a classic case of fractal. It has infinitely fine structure levels and strict self-similar characteristics. It is very helpful for people to understand the fractal idea vividly, and the drawing method is very simple and interesting.

Briefly explain the process of drawing the Sierpinski triangle: First, let $\mathrm{S}_{0}$ be an equilateral triangle with a side length of 1 , first step $(n=1)$, connect the midpoints of the three sides to get four congruent small triangles, remove the middle one Small triangle, keep the remaining three small triangles to get $S_{1}$. In the second step $(n=2)$, repeat the previous step for the remaining three small triangles in $S_{1}$ to obtain $S_{2}$, which contains nine small triangles. By repeating the above steps in this way, $\mathrm{S}_{\mathrm{n}}$ can be obtained. When $\mathrm{n}$ approaches infinity, a Sierpinski triangle is obtained. Fig 1 shows the formation process of the Sierpinski triangle. 


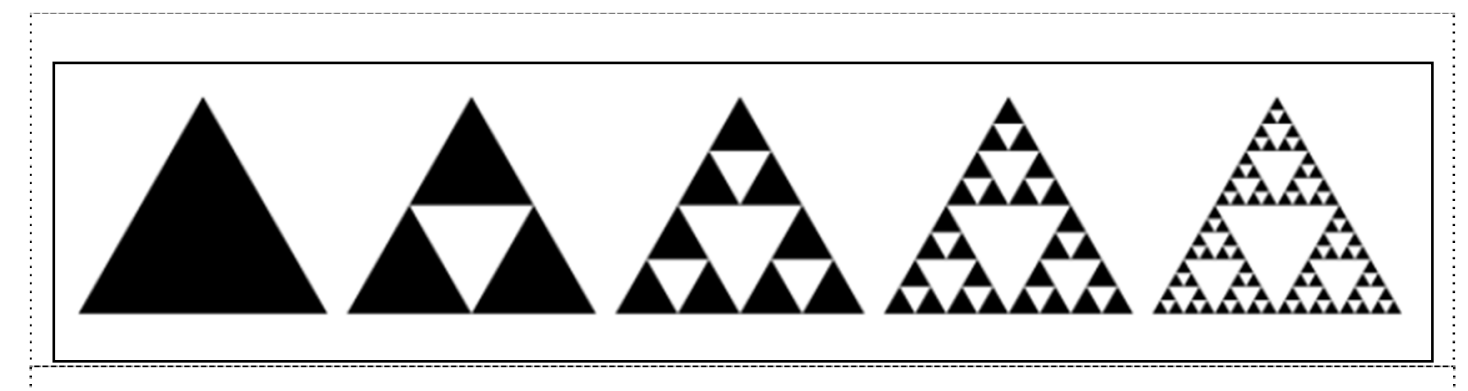

Fig 1. The formation of the Sierpinski triangle.

Because of the self-similarity of fractals, fractal patterns are infinite in both depth and breadth. At the same time, fractal art patterns are created with the help of computers, so that they can surpass the human brain to a certain extent. Thinking, therefore, the degree of randomness and arbitrariness of its pattern is relatively large, but this will often produce unexpected effects (new, chic, and strange), showing people a refreshing feeling and a strong sense of the times. Fractals are ubiquitous in nature and can be artificially produced. Typical fractals in daily life include ferns, galaxies, broccoli, and the blood vessels of the coastline and even the human brain.

\subsection{Aesthetic characteristics of fractal art patterns}

The colorful fractal art pattern has an undisputed visual shock. With science or aesthetics, people have new feelings when they first see the fractal art pattern. Although the fractal art pattern is a computer graphic generated by the idea of chaotic fractal using a computer programming language, it shows a new artistic form different from the traditional form beauty. Its aesthetic characteristics are as follows:

1.3.1. Balance. The balance in the fractal art pattern is a kind of dynamic balance, but it is different from the symmetry in the traditional sense. It is a balance where the whole and the part are mutually restrained, and the overall visual state is relatively felt. It is this balanced form that makes the fractal pattern more conducive to showing the overall effect of stability, liveliness, dexterity and order. The complexity of the fractal pattern and the infinitely fine structure are its typical characteristics. Although it is complex, it has a balanced visual relationship, and the beauty is self-evident. As shown in Fig 2, the self-similar characteristics of the fractal pattern allow it to have non-unique center points. Multiple center points are scattered uniformly in order and restrict each other, thereby avoiding visual conflicts and showing a dynamic and balanced vision.

1.3.2. Harmonious. The fractal art pattern has a mathematically harmonious expression, but this harmonious expression has got rid of the indifference and monotony of traditional European geometry, so that the fractal pattern can be based on the identity and supplemented by the fusion of differences. In this way, each local shape change and each color's situation echo each other. The transition is natural and there is no bluntness. The organic change and unity of this form is harmony. As shown in Fig 3, the local pattern of the first level of the fractal pattern serves as a local feature of the second level, so that the overall situation has an excessive relationship that is not intended to be natural, and enhances the overall sense of harmony.

1.3.3. Symmetry. Symmetric structure is one of the typical characteristics of fractal. It is a new form of symmetry that is different from up and down and left and right. This symmetry does not bring a monotonous and rigid feeling like the symmetry of traditional europeanstyle geometric forms, but the entire fractal pattern can contain dynamic beauty in the balance, making people excited and excited. It can also show rigorous correspondence and restrictions, so that this wild freedom will not lose control and tend to be chaotic[3]. As shown in Fig 4, the fractal pattern, while inheriting the symmetry of the traditional form, also broke through its existing expression. It can be said that it is a local and larger part symmetry. Any small-scale part maintains the shape with the whole Similar, to achieve a harmonious and balanced visual experience.

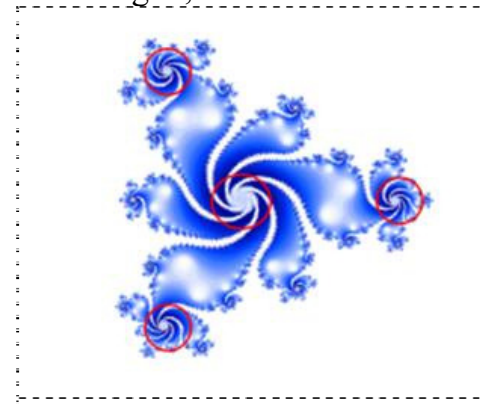

Fig 2. Balance in fractal.

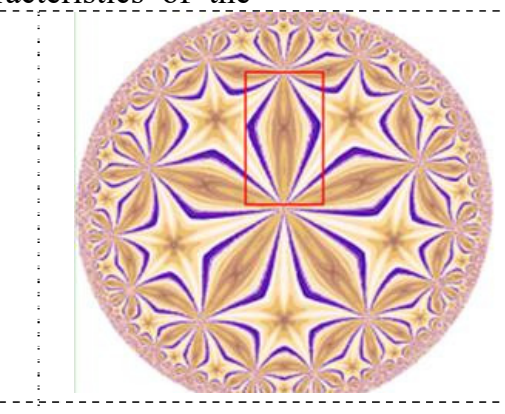

Fig 3. The fractal in harmony.

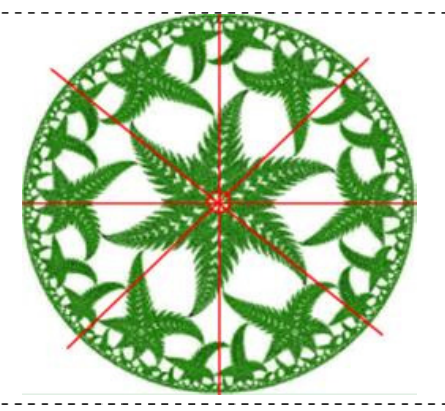

Fig 4. The fractal in symmetrical. 
1.3.4. Self-similarity. This self-similarity of fractals makes fractal patterns infinite in both depth and breadth. At the same time, fractal art patterns are created with the help of computers, so that it can surpass the thinking and imagination of the human brain to a certain extent., So its pattern has great randomness and arbitrariness, but often it is unexpectedly novel, chic, peculiar, and varied,

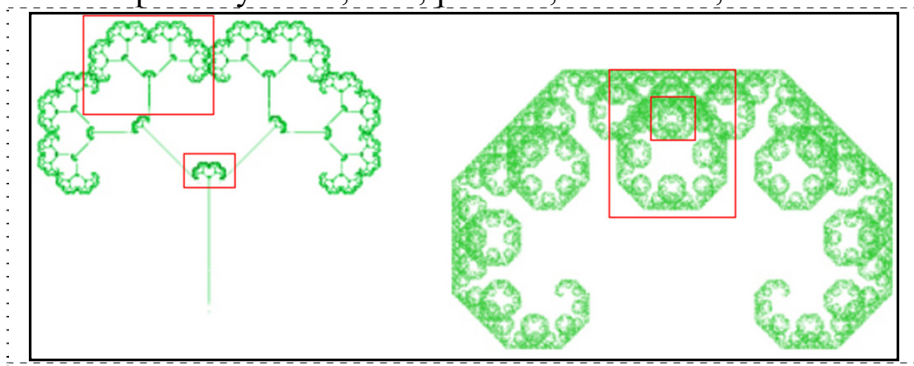

Fig 5. The fractal's self-similarity. and can achieve refreshing visual effects. The fractal tree and Lévy curve shown in Fig 5 are typical fractal structures. If the part is infinitely enlarged, a small fractal tree similar to the whole and a reduced version of the Levi curve can be observed.
1.3.5. Order in chaos. The impression of fractal art patterns is often complex and mysterious. However, the aesthetic ideal pursued by fractal patterns is neither simple nor complicated, but an order in chaos, seeking richness in unity. As Gombrich said, "Aesthetic pleasure comes from watching a pattern between tedious and messy; monotonous patterns are difficult to attract burden our perception system. Too heavy to stop viewing it [4]. "The structure of the fractal pattern is complicated, it always has an endless winding inside, as shown in the Julia set shown in Fig 6, the pattern has an infinitely fine structure, each part There are more changes in the edge details. However, the entire pattern is not messy. The change of clustering and scattering, sparseness and denseness is particularly appropriate. It contains an internal order, symmetry with the whole.

\section{Selection and analysis of fractal patterns in ceramic design practice}

Decorative pattern is an important artistic expression of ceramic design, which emphasizes aesthetic principles

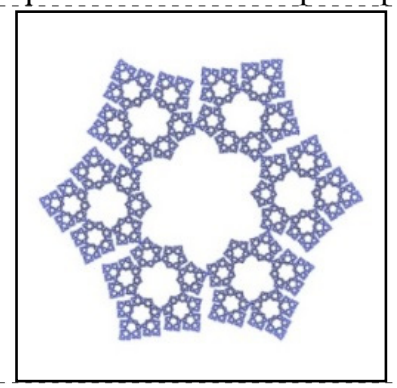

Fig 7. Z6 symmetrical fractal pattern.

\subsection{Design concept} people's attention, and overly complex patterns will

\section{Conception and details of design practice}

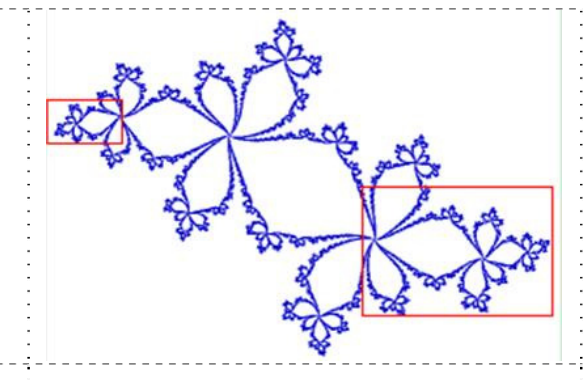

Fig 6. Julia set. such as balance, symmetry, harmony and unity. The computer-generated fractal art pattern is magical, beautiful, and unpredictable. It has self-similarity and infinite fineness. The structure contains new aesthetic expressions beyond traditional aesthetics.

The fractal pattern shown in Fig 7 is a $Z_{6}$ symmetric fractal generated by a non-linear iterative function system constructed with six parameters with a symmetrical distribution selected in the 1-period attraction parameter area of a symmetric generalized $M$ set of complex polynomials by computer technology 7 pttern [5]. The pattern has the following characteristics: the pattern is six-element rotationally symmetric; if the six vertices are connected, a regular hexagon can be formed; it has an infinitely fine structure level; the part and the whole, the part and smaller parts have symmetry and self-similarity; The pattern starts from the center point of the overall figure, and the parts of each level remain similar to the overall shape. And it is a dynamic symmetry, which is a typical feature of a fractal pattern. This symmetry is different from the traditional symmetry. It is the symmetry between the local and the whole of the figure. This dynamic symmetry gives a harmonious beauty.

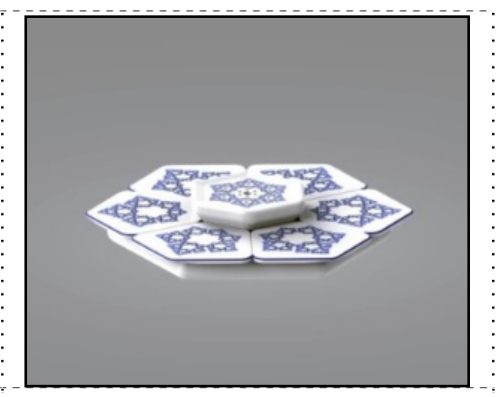

Fig 8. Fractal teaboard.

Inspired by the fractal pattern shown in Fig 7, a hexagonal fractal tea tray was designed, as shown in Fig 8. According to the shape and structural characteristics shown in Fig 7, a regular hexagon is selected as the overall outline of the tea tray, and six deformed small 
hexagon structures with self-similar structural characteristics are used as the dividing nodes of the six trays according to the first level of the pattern; It was artistically and functionally increased and decreased, and the arrangement and layout were adjusted, mainly to express its meaning rather than copy its shape, so that the fractal pattern is in harmony with the shape of the tea tray. Show aesthetic characteristics such as rhythm and self-similarity.

\subsection{Plan details}

The fractal pattern shown in Fig 7 is a six-unit rotational symmetry. From the perspective of the figure, it rotates outwards around the center point, and a deformed secondary pattern similar to the overall structure appears in each of the six apex directions. In this design, the center of the fractal pattern is used as the center of the tea tray, and the six vertices of the pattern are connected by straight lines to form a regular hexagon as the outer contour of the base of the tea tray. As shown in Fig 9, the middle of the tea tray base is a regular hexagonal boss, the shape matches the overall structure of the fractal pattern. The center of the tea tray boss coincides with the center of the base, and the corresponding positions of the

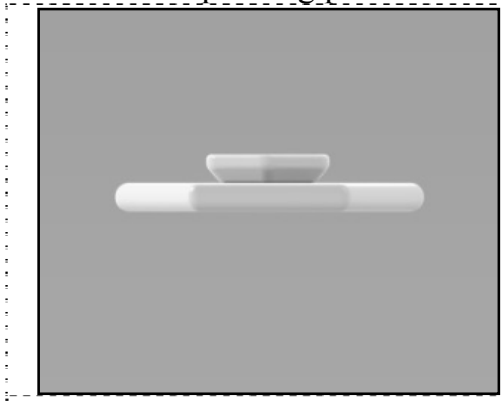

Fig 9. The vertical view of the fractal teaboard base.

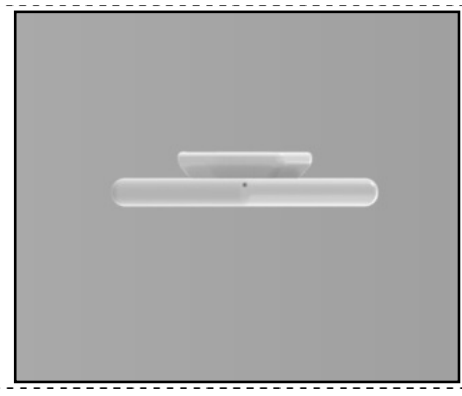

Fig 11. The left view of the fractal teaboard.

The tea tray base is composed of six pentagonal trays. The shape characteristics are derived from the shape and structural characteristics of the fractal pattern, and its arrangement and layout are adjusted at the same time. According to the characteristics of the fractal pattern shown in Fig 7, the first six levels of the deformed small hexagonal structure with self-similar structural characteristics are used as the division nodes of the six trays; without destroying the overall fractal pattern, two regular hexagons are arranged at a 30-degree angle to each other. The tea tray boss is in the shape of an inverted six-sided platform, seen from the front as a pure lotus, as shown in Fig 10. The top of the tray of the tea tray can be used for placing the teapot. The fractal pattern shown in Fig. 7 is scaled according to an appropriate proportion. The six corners of the pattern correspond to the six apex directions of the hexagonal boss and match the top surface of the boss. According to the structural characteristics of the fractal pattern, 7 circular drainage holes are arranged at the center of the top surface of the boss of the tea tray, arranged in a regular hexagon, corresponding to the directions of the six vertices of the boss, and in a small level similar to the overall structure There is also a circular drainage hole at the center of the pattern, which is used to discharge waste water. At the same time, the top surface of the boss is concave, so that the situation of tea overflowing can be avoided. The inside of the tray base is hollow, as a storage space for waste water. Seven circular drainage holes are arranged in a corner of the tray base, arranged in a regular hexagon, and can be dumped at any time to drain the water in the tray base, as shown in the Fig 11 is shown.

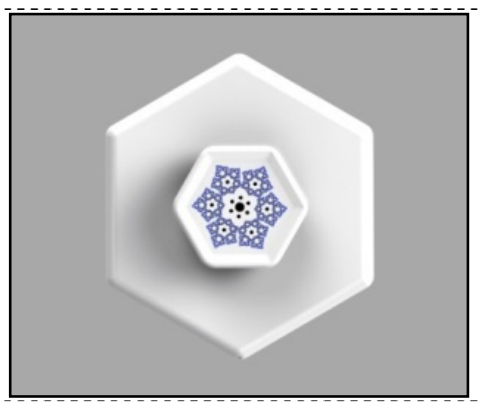

Fig 10. The front view of the fractal teaboard.

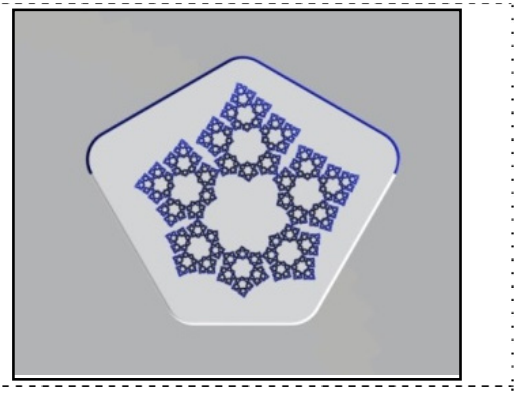

Fig 12. The vertical view of the fractal tea tray.

divided into six partial patterns of the same size and similar to the overall pattern shape, and sequentially connecting the six vertices of the partial pattern to obtain a small hexagonal pattern. Considering that the fractal pattern and the shape of the tea tray can be properly matched, the shape of the tray is first designed as a hexagon, the six trays form a complete, similarly shaped large hexagon. Because the tea tray is a convex table shaped like an inverted hexagon, the design needs to use 
the principle of subtraction and cut out the area occupied by the boss, that is, the shape of the final tray is designed as a pentagon. Fig 12 shows a top view of the tray. The six self-similar patterns of the first level of the fractal pattern are scaled according to the appropriate proportions, centered on the top surface of the tray, make sure the direction of the pattern apex is the same as the direction of the top corner of the tray. The six trays are arranged in sequence, and together with the tray base, a

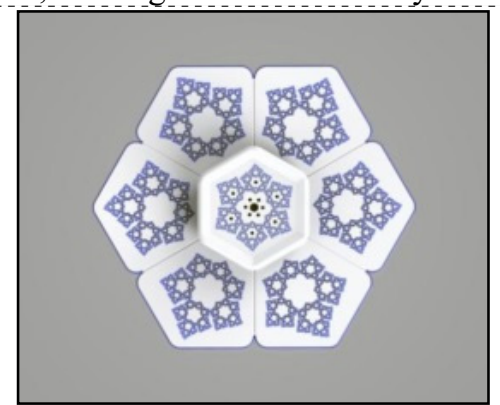

Fig 13. The vertical view teaboard. complete tea is formed. Fig 13 is a top view of the tea tray design. The two outer contour edges of the six trays are dotted with blue lines. The 12 blue lines are connected to each other, highlighting the overall modeling vitality of the tea tray. From a distance, the tea tray is in full bloom in the pond. A lotus plant is fresh and elegant, bringing a natural and peaceful atmosphere to people.

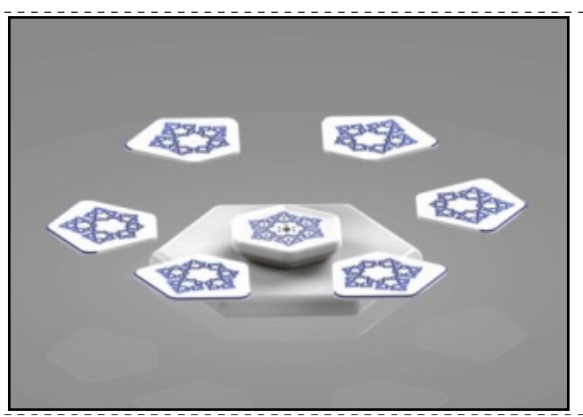

Fig 14. The explosion figure of the teaboard.

simple and clear. The outer surface of the tea cup is designed as a partial pattern of $1 / 6$ of the fractal pattern. The pattern is rotated and scaled to a certain angle to form a connection and echo relationship with the tea cup tray pattern. At the same time, it breaks the original indifferent geometry and adds some life taste. The teapot is placed on the upper surface of the middle boss of the tea plate, and the corresponding positions on the outer surface of the outer plate are designed with a fractal pattern consistent with the tray, which produces a good sense of stability. As shown in Fig 16, this combination of teapot and cup natural, harmonious, connected and unified.

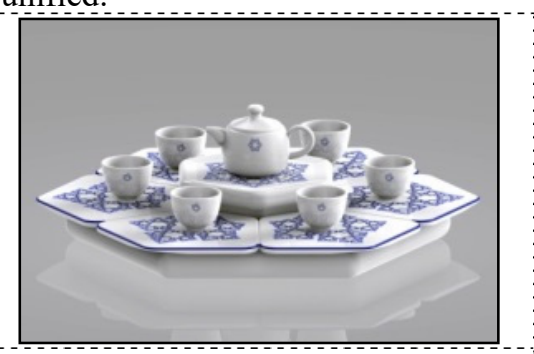

Fig 16. The picture of the fractal tea set.

The hexagonal fractal ceramic tea set is suitable for being placed in the living room, dining room, study, courtyard, office. It can be used for tea drinking and tea tasting, as well as ornamental function. The main body and parts of the tea set reflect the fractal idea, and the shape and decoration complement each other, which not only meets the functional needs of people, but also meets the aesthetic needs of people.

\section{Conclusion}

Fractal geometry reveals a kind of "real" geometry that is closer to the original appearance of nature and can better show the inner structure of nature. It is called "natural geometry" [6]. Applying it to product design can give the 
product its natural essence, showing an evolutionary and morphological characteristic of the dynamic growth of nature.

Use fractal ideas to guide product design and creation, and apply fractal patterns to the design and research of ceramic tea sets, which can better express the form beauty and decorative design elements of ceramic tea, thereby designing a more humane and able to bring people ceramic ware that has a stronger visual beauty. Fractal ideas can provide new inspiration for product design, expand product design ideas, and inspire more possibilities. Through the use of fractal ideas and fractal patterns, the design can reflect infinitely local and overall self-similar structural characteristics, providing a new modeling language for product design. The effective application of fractal ideas in the field of design has also promoted the development of the concept of fractal, broadened the application field of fractal disciplines, and has practical significance and practical application value. The two promote each other and develop together.

\section{References}

1. Mandelbrot, B.B. (1986) The fractal geometry of nature. W. H. Freeman and Co., San Francisco.

2. Mandelbrot, B.B.(1987) Fractal. Encyclopedia of Physical Science and Technology.

3. Zhu.W.Y. (2003) Research and Application of Complex Map M-J Fractal Spectrum Scale Invariance and Fractal Dimensional Information Symmetry, Northeast University,Shenyang.

4. Xia.L.L. (2011) Research on the application of digital graphics in fashion design based on fractal geometry theory. Zhejiang sci-tech university,Hangzhou.

5. Feng.D.D. (2016) Research on nonlinear IFS iterative gunction system constructed from complex analytic map. Shenyang Jianzhu University,Shenyang.

6. Zhao.Y.P. (2003) Application of fractal geometry in architecture. Dalian University of Technology,Dalian. 